\title{
Fixed-point Theorems in G-complete Fuzzy Metric Spaces
}

\author{
Naser Abbasi, Hamid Mottaghi Golshan, Mahmood Shakori
}

Department of Mathematics, Lorestan University, Khoramabad, Iran

Email address:

naserabbasi persia@yahoo.com (N. Abbasi),motgolham@gmail.com (H. M. Golshan), mahmoodshakoori@gmail.com (M. Shakori)

\section{To cite this article:}

Naser Abbasi, Hamid Mottaghi Golshan, Mahmood Shakori. Fixed-point Theorems in G-complete Fuzzy Metric Spaces. Pure and Applied Mathematics Journal. Vol. 4, No. 4, 2015, pp. 159-163. doi: 10.11648/j.pamj.20150404.14

\begin{abstract}
In the present paper we introduce generalized contraction mapping in fuzzy metric space and some fixed-point theorems for G-complete fuzzy metric space are proved. Our results generalize and extend many known results in metric spaces to a (non-Archimedean) fuzzy metric space in the in the sense of George and Veeramani [George A, Veeramani P, On some results in fuzzy metric spaces, Fuzzy Sets and Systems 1994;64:395-9].
\end{abstract}

Keywords: Fuzzy Metric Spaces, Generalized Contraction Mapping, G-Complete

\section{Introduction}

Kramosil and Michalek [7] generalized the concept of probabilistic metric space and studied an interesting notion of fuzzy metric space with the help of continuous t-norm. Later on, in order to construct a Hausdorff topology on the fuzzy metric space, George and Veeramani [4] modied the concept of fuzzy metric space introduced by Grabiec [5] and Kramosil and Michalek [7]. Fixed point theory has important applications in diverse disciplines of mathematics, statistics, engineering and economics in dealing with problems arising in: approximation theory, potential theory, game theory, mathematical economics, etc. Fixed-point theory for contraction type mappings in fuzzy metric space is closely related to the fixed-point theory for the same type of mappings in probabilistic metric space of Menger type (see $[9,13])$. Many authors $[3,5,6,8,11]$ have proved some fixed point theorems for various generalizations of contraction mappings in probabilistic and fuzzy metric spaces. In this paper we discuss generalized contraction mapping in fuzzy metric space in the sense George and Veeramani [4]. To de ne the fuzzy metric space we have to state several concepts as follows:

Definition $1.1([12])$. A binary operation $*$ is continuous t-norm if satisfies the following conditions:
(a) * is commutative and associative;
(b) $*$ is continuous;
(c) $a * 1=a$ for all $a \in[0,1]$;
(d) $a * b \leq c * d \quad$ whenever $a \leq c$ and $b \leq d$ and $a, b, c, d \in[0,1] \cdot$

The t-norm minimum will be denoted by min, the usual product by. These two t-norms are the most commonly used in fuzzy logic.

Definition 1.2 ([4]). A fuzzy metric space is an ordered triple $(X, M, *)$ such that $X$ is a nonempty set, $*$ is a continuous t-norm and $M$ is a fuzzy set of $X \times X \times(0,1)$ satisfying the following conditions, for all $x, y, z \in X, s, t>0$ :

(FM1) $M(x, y, t)>0$;

(FM2) $M(x, y, t)=1$ if and only if $x=y$;

(FM3) $M(x, y, t)=M(y, x, t)$;

(FM4) $M(x, z, t+s) \geq M(x, y, t) * M(y, z, s)$;

(FM5) $M(x, y, \cdot):(0,+\infty) \rightarrow[0,1]$ is continuous.

If, in the above definition, the triangular inequality (FM4) is replaced by (NAF)

$$
M(x, z, t) \geq M(x, y, t) * M(y, z, t), \forall x, y, z \in X, \forall t>0,
$$

then the triple $(X, M, *)$ is called a non-Archimedean fuzzy metric space.

George and Veeramani [4] proved that every fuzzy metric space $(X, M, *)$ on $X$ generates a Hausdorff first countable topology $\tau_{M}$ on $X$ which has as a base the family of sets of the form $\left\{B_{M}(x, \epsilon, t): x \in X, 0<\epsilon<1, t>0\right\}$, where $B_{M}(x, r, t)=\{y \in X: M(x, y, t)>1-r\}$ for all $r \in(0,1), t>0$.

Remark 1.3 ([4]). In fuzzy metric space $(X, M, *)$, 
$M(x, y, \cdot)$ is non decreasing for all $x, y \in X$.

Definition 1.4 ([5]). A sequence $x_{n}$ in $X$ is said to be convergent to a point $\mathrm{x}$ in $X$ (denoted by $x_{n} \rightarrow x$ ), if $M\left(x_{n}, x, t\right) \rightarrow 1$,for all $t>0$.

Definition 1.5 ([4]). (a) A sequence $x_{n}$ in a fuzzy metric space $(X, M, *)$ is a Cauchy sequence if for each $\epsilon \in(0,1)$ and each $t>0$ there exists $n_{0} \in \mathbb{N}$ such that $M\left(x_{n}, x_{m}, t\right)>1-\epsilon$ for all $n, m \geq n_{0}$.

(b) We say fuzzy metric space $(X, M, *)$ is complete if every Cauchy sequence is convergent with respect to $\tau_{M}$.

Remark $1.6([10])$. Let $(X, M, *)$ be a fuzzy metric space then $M$ is a continuous function on $X \times X \times(0,1)$.

Example 1.7. Let $(X, d)$ be an ordinary metric space and * be a t-norm.

(1) (See [4]) Let $M_{d}$ be a fuzzy set on $X \times X \times(0,1)$ define as follow:

$$
M_{d}(x, y, t)=\frac{h t^{n}}{h t^{n}+m d(x, y)}
$$

for all for all $t, h, m, n \in \mathbb{R}^{+}$. Then $\left(X, M_{d}, *\right)$ is a fuzzy metric space and called induced fuzzy metric space. If in equation (1.1) we take $h=m=n=1$ then we have

$$
M_{d}(x, y, t)=\frac{t}{t+d(x, y)} .
$$

This fuzzy metric space $\left(X, M_{d}, *\right)$ is called standard fuzzy metric space.

(2) (See [11]) It is immediate to show that $(X, d)$ is a non-Archimedean metric space if and only if $\left(X, M_{d}, \mathrm{~min}\right)$ is a non-Archimedean fuzzy metric space.

Further examples and results for fuzzy metric spaces may be found in $[4,5,7,10,12]$.

\section{Main Results}

Gregori and Sepena introduced the notions of fuzzy contraction mapping and fuzzy contractive sequence as follows:

Definition 2.1 ([6]). Let $(X, M, *)$ be a fuzzy metric space. We call the the mapping $T: X \rightarrow X$ is fuzzy contractive mapping, if there exists $\lambda \in(0,1)$ such that

$$
\frac{1}{M(T x, T y, t)}-1 \leq \lambda\left(\frac{1}{M(x, y, t)}-1\right)
$$

for each $x, y \in X$ and $t>0,(\lambda$ is called the contractive constant of $T$ ).
Recall that a sequence $x_{n}$ in a metric space $(X, d)$ is said to be contractive if there exists $\lambda \in(0,1)$ such that $d\left(x_{n}, x_{n+1}\right) \leq \lambda d\left(x_{n-1}, x_{n+1}\right)$, for all $n \in \mathbb{N}$.

Definition 2.2 ([6]). Let $(X, M, *)$ be a fuzzy metric space. A sequence $x_{n}$ is called fuzzy contractive if there exists $\lambda \in(0,1)$ such that

$$
\frac{1}{M\left(x_{n}, x_{n+1}, t\right)}-1 \leq \lambda\left(\frac{1}{M\left(x_{n-1}, x_{n}, t\right)}-1\right)
$$

for every $t>0, n \in \mathbb{N}$.

Definition 2.3 ([6]). Let be $(X, M, *)$ a fuzzy metric space. A sequence is called $x_{n}$ G-Cauchy if for each $t>0$ and $p \in \mathbb{N}, \lim _{n \rightarrow \infty} M\left(x_{n+p}, x_{n}, t\right)=1$.

A fuzzy metric space in which every G-Cauchy sequence is convergent is called G-complete.

The following proposition is justified the above definitions.

Proposition 2.4 ([6]). (a) The sequence $x_{n}$ in the metric space $(X, d)$ is contractive iff $x_{n}$ is fuzzy contractive in the induced fuzzy metric space $\left(X, M_{d}, *\right)$.

(b) The standard fuzzy metric space $\left(X, M_{d}, *\right)$ is complete iff the metric space $(X, d)$ is complete.

(c) If sequence $x_{n}$ is fuzzy contractive in $\left(X, M_{d}, *\right)$ then it is G-Cauchy.

A continuous t-norm $*$ is of Hadžić-type if there exists a strictly increasing sequence $b_{n} \in(0,1)$ such that $b_{n} * b_{n}=b_{n}$ for all $n \in \mathbb{N}, *=\min$ is an example of such t-norm.

Lemma 2.5 ([8]). Each complete non-Archimedean fuzzy metric space $(X, M, *)$ with $*$ of Hadžić-type is G-complete.

Let we recall the generalized contractions mapping in metric spaces due to Ćirić [1].

Theorem 2.6. Let $(X, d)$ be a complete metric space and $T: X \rightarrow X$ be a self-mapping on $X$ such that for each Equation Chapter (Next) Section $2 x, y \in X$.

$$
\begin{aligned}
d(T x, T y) \leq & \alpha(x, y) d(x, y)+\beta(x, y) d(x, T x)+\gamma(x, y) d(y, T y) \\
& +\delta(x, y)\left[\frac{d(x, T y)+d(y, T x)}{2}\right]
\end{aligned}
$$

where $\alpha, \beta, \gamma, \delta$ are functions from $X \times X$ into $[0,1)$ such that

$$
\begin{aligned}
& \lambda=\sup \{\alpha(x, y)+\beta(x, y) \\
& +\gamma(x, y)+\delta(x, y) ; x, y \in X\}<1
\end{aligned}
$$

Then $T$ has a unique fixed point.

Mapping which satisfies (2.1) called generalized contractions. As observed in [1], a self-mapping $T$ on a 
metric space $(X, d)$ is a generalized contraction if and only if $T$ satisfies the following condition:

$$
\begin{gathered}
d(T x, T y) \leq \lambda \max \{d(x, y), d(x, T x), d(y, T y), \\
\left.\frac{d(x, T y)+d(y, T x)}{2}\right\}
\end{gathered}
$$

where. Later on Ćirić take the term $\max \{d(x, T y), d(y, T x)\}$ instead of $[d(x, T y)+d(y, T x)] / 2$ and by a new method of proof deduce theorem 2.6 for following which he called it quasicontraction mapping

$$
\begin{gathered}
d(T x, T y) \leq \lambda \max \{d(x, y), d(x, T x), d(y, T y), \\
d(x, T y), d(y, T x)\}
\end{gathered}
$$

The following is quasi-contraction theorem for non-Archimedean fuzzy metric spaces:

Theorem 2.7. Let $(X, M, *)$ be a G-complete fuzzy metric space where the continuous t-norm $*$ is defined as min and $T: X \rightarrow X$ be self-mapping on $X$ such that for each $x, y \in X, t>0$

$$
\begin{aligned}
& \frac{1}{M(T x, T y, t)}-1 \leq \alpha\left(\frac{1}{M(x, y, t)}-1\right) \\
& +\beta\left(\frac{1}{M(x, T x, t)}-1\right)+\gamma\left(\frac{1}{M(y, T y, t)}-1\right) \\
& +\delta\left(\frac{1}{M(x, T y, 2 t)}+\frac{1}{M(y, T x, 2 t)}-2\right),
\end{aligned}
$$

Proof. Let $x \in X$ and $t>0$ be arbitrary and consider a sequence of Pickard iterations $x_{n}$, defined inductively by $x_{0}=x, x_{1}=x_{0}, \ldots, x_{n+1}=T\left(x_{n}\right)$ for each $n=\mathbb{N}$, we will show that $x_{n}$ is fuzzy contractive. From (2.4) by replacing $x=x_{n-1}, y=x_{n}$ we get

$$
\begin{aligned}
& \frac{1}{M\left(x_{n}, x_{n+1}, t\right)}-1=\frac{1}{M\left(T x_{n-1}, T x_{n}, t\right)}-1 \\
& \leq \alpha\left(\frac{1}{M\left(x_{n-1}, x_{n}, t\right)}-1\right)+\beta\left(\frac{1}{M\left(x_{n-1}, x_{n}, t\right)}-1\right) \\
& +\gamma\left(\frac{1}{M\left(x_{n}, x_{n+1}, 2 t\right)}-1\right)+\delta\left(\frac{1}{M\left(x_{n-1}, x_{n+1}, 2 t\right)}-1\right) .
\end{aligned}
$$

By our choice of t-norm $*$ and triangular inequality for the last parenthesis in (2.5) we have

$$
\begin{aligned}
& \frac{1}{M\left(x_{n-1}, x_{n+1}, 2 t\right)}-1 \leq \\
& \frac{1}{\min \left\{M\left(x_{n-1}, x_{n}, t\right), M\left(x_{n}, x_{n+1}, t\right)\right\}}-1= \\
& \max \left\{\frac{1}{M\left(x_{n-1}, x_{n}, t\right)}-1, \frac{1}{M\left(x_{n}, x_{n+1}, t\right)}-1\right\}
\end{aligned}
$$

from (2.5) we get

$$
\begin{aligned}
& \frac{1}{M\left(x_{n}, x_{n+1}, t\right)}-1 \leq \\
& (\alpha+\beta+\gamma) \max \left\{\frac{1}{M\left(x_{n-1}, x_{n}, t\right)}-1, \frac{1}{M\left(x_{n}, x_{n+1}, t\right)}-1\right\}
\end{aligned}
$$

hence,

$$
\begin{aligned}
& \frac{1}{M\left(x_{n}, x_{n+1}, t\right)}-1 \leq \lambda \max \left\{\frac{1}{M\left(x_{n-1}, x_{n}, t\right)}-1\right. \\
& \left., \frac{1}{M\left(x_{n}, x_{n+1}, t\right)}-1\right\}
\end{aligned}
$$

where $\lambda<1$, this implies

$$
\frac{1}{M\left(x_{n}, x_{n+1}, t\right)}-1 \leq \lambda\left(\frac{1}{M\left(x_{n-1}, x_{n}, t\right)}-1\right)
$$

so sequence $x_{n}$ is fuzzy contractive sequence. Since $(X, M, *)$ is a complete fuzzy metric space so by proposition 2.4 and lemma 2.5, sequence $x_{n}$ converges to $u$ for some $u \in X$. Now we show $u$ is fixed point of $T$, from (2.4) we have

$$
\begin{aligned}
& \frac{1}{M\left(T u, T x_{n}, t\right)}-1 \leq \alpha\left(\frac{1}{M\left(u, x_{n}, t\right)}-1\right)+ \\
& \beta\left(\frac{1}{M(u, T u, t)}-1\right)+\gamma\left(\frac{1}{M\left(x_{n}, x_{n+1}, t\right)}-1\right) \\
& +\delta\left(\frac{1}{M\left(u, x_{n+1}, 2 t\right)}-1\right)+\eta\left(\frac{1}{M\left(x_{n}, T u, 2 t\right)}-1\right),
\end{aligned}
$$

taking the limit as $n \rightarrow+\infty$ we obtain

$$
\begin{aligned}
& \frac{1}{M(T u, u, t)}-1 \leq \beta\left(\frac{1}{M(u, T u, t)}-1\right) \\
& +\eta\left(\frac{1}{M(u, T u, t)}-1\right) \leq \lambda\left(\frac{1}{M(u, T u, t)}-1\right)
\end{aligned}
$$

Since $\lambda<1$, we have $M(u, T u, t)=1$, thus, $T u=u$, by (2.9) we find fixed point is unique.

Theorem 2.8. Let $X$ be a complete non-Archimedean fuzzy metric space where the continuous t-norm $*$ is defined as min and $T: X \rightarrow X$ be a self-mapping on $X$ such that for each $x, y \in X, t>0$.

$$
\begin{aligned}
& \frac{1}{M(T x, T y, t)}-1 \leq \alpha\left(\frac{1}{M(x, y, t)}-1\right) \\
& +\beta\left(\frac{1}{M(x, T x, t)}-1\right)+\gamma\left(\frac{1}{M(y, T y, t)}-1\right) \\
& +\delta\left(\frac{1}{M(x, T y, t)}-1\right)+\eta\left(\frac{1}{M(y, T x, t)}-1\right),
\end{aligned}
$$


where $\alpha, \beta, \gamma, \delta, \eta \in[0,1)$ and $\lambda=\alpha+\beta+\gamma+\delta+\eta<1$, Then $T$ has a unique fixed point.

Proof. The proof is very similar to the theorem 2.8. In stead of the equation (2.5) and (2.6) we find

$$
\begin{aligned}
& \frac{1}{M\left(x_{n}, x_{n+1}, t\right)}-1=\frac{1}{M\left(T x_{n-1}, T x_{n}, t\right)}-1 \\
& \leq \alpha\left(\frac{1}{M\left(x_{n-1}, x_{n}, t\right)}-1\right)+\beta\left(\frac{1}{M\left(x_{n-1}, x_{n}, t\right)}-1\right) \\
& +\gamma\left(\frac{1}{M\left(x_{n}, x_{n+1}, t\right)}-1\right)+\delta\left(\frac{1}{M\left(x_{n-1}, x_{n+1}, t\right)}-1\right) .
\end{aligned}
$$

and

$$
\begin{aligned}
& \frac{1}{M\left(x_{n-1}, x_{n+1}, t\right)}-1 \leq \\
& \frac{1}{\min \left\{M\left(x_{n-1}, x_{n}, t\right), M\left(x_{n}, x_{n+1}, t\right)\right\}}-1= \\
& \max \left\{\frac{1}{M\left(x_{n-1}, x_{n}, t\right)}-1, \frac{1}{M\left(x_{n}, x_{n+1}, t\right)}-1\right\},
\end{aligned}
$$

respectively. Proceed as the proof of the Theorem 2.8 then we find sequence $x_{n}$ is fuzzy contractive, Since $(X, M, *)$ is a complete fuzzy metric space so by proposition 2.4 and lemma 2.5 there exists $u \in X$ such that $\lim _{n \rightarrow \infty} x_{n}=u$. Instead of (2.8) we find

$$
\begin{aligned}
& \frac{1}{M\left(T u, T x_{n}, t\right)}-1 \leq \alpha\left(\frac{1}{M\left(u, x_{n}, t\right)}-1\right)+ \\
& \beta\left(\frac{1}{M(u, T u, t)}-1\right)+\gamma\left(\frac{1}{M\left(x_{n}, x_{n+1}, t\right)}-1\right) \\
& +\delta\left(\frac{1}{M\left(u, x_{n+1}, t\right)}-1\right)+\eta\left(\frac{1}{M\left(x_{n}, T u, t\right)}-1\right),
\end{aligned}
$$

taking the limit as $n \rightarrow \infty$ we obtain

$$
\begin{aligned}
& \frac{1}{M(T u, u, t)}-1 \leq \beta\left(\frac{1}{M(u, T u, t)}-1\right) \\
& +\eta\left(\frac{1}{M(u, T u, t)}-1\right) \leq \lambda\left(\frac{1}{M(u, T u, t)}-1\right) .
\end{aligned}
$$

Since $\lambda<1$, we have $M(u, T u, t)=1$, thus, $T u=u$, by (2.9) we find fixed point is unique.

Remark 2.9. As observed in [2], by a similar proof we find the generalized contraction condition (2.4) and (2.9) are equivalent to following:

$$
\frac{1}{M(T x, T y, t)}-1 \leq \lambda \max \left\{\left(\frac{1}{M(x, y, t)}-1\right),\right.
$$

$$
\begin{aligned}
& \left(\frac{1}{M(x, T x, t)}-1\right),\left(\frac{1}{M(y, T y, t)}-1\right), \\
& \left.\left(\frac{1}{M(x, T y, t)}-1\right),\left(\frac{1}{M(y, T x, t)}-1\right)\right\}
\end{aligned}
$$

and

$$
\begin{aligned}
& \frac{1}{M(T x, T y, t)}-1 \leq \lambda \max \left\{\left(\frac{1}{M(x, y, t)}-1\right),\right. \\
& \left(\frac{1}{M(x, T x, t)}-1\right),\left(\frac{1}{M(y, T y, t)}-1\right), \\
& \left.\left(\frac{1}{M(x, T y, 2 t)}-1\right),\left(\frac{1}{M(y, T x, 2 t)}-1\right)\right\}
\end{aligned}
$$

respectively, where ${ }^{\lambda \in(0,1)}$.

Remark 2.10. For complete metric space $(X, d)$, consider standard fuzzy metric space $\left(X, M_{d}, *\right)$ in example 1.7 then by the following equality

$$
\frac{1}{M(x, y, t)}-1=\frac{d(x, y)}{t}
$$

and above remark we find contractions (2.2) and (2.4) are equivalent. Also by proposition 2.4-(a) and (b) and following the same line as Theorem 2.7, we automatically deduce fuzzy contractive sequence (2.7) is convergent, so theorem 2.6 is special case of Theorem 2.7. In next theorem the assumption $*=\min$ in theorem 2.7 is not needed.

Theorem 2.11. Let $(X, M, *)$ be a G-complete fuzzy metric space and $T: X \rightarrow X$ be a self mapping on $X$ such that for each $x, y \in X, t>0$

$$
\begin{aligned}
& \frac{1}{M(T x, T y, t)}-1 \leq \alpha\left(\frac{1}{M(x, y, t)}-1\right) \\
& +\beta\left(\frac{1}{M(x, T x, t)}-1\right)+\gamma\left(\frac{1}{M(y, T y, t)}-1\right),
\end{aligned}
$$

where $\alpha, \beta, \gamma \in[0,1)$ and $\lambda=\alpha+\beta+\gamma<1$. Then $T$ has a unique fixed point.

Proof. The assumption t-norm $*=\min$ in theorem 2.7 is applied only in equation (2.6), so the proof is very similar to theorem 2.7 and it is omitted.

Corollary 2.12. Let $\left(X, M_{d}, *\right)$ be a complete standard fuzzy metric space and $T: X \rightarrow X$ be a self-mapping on $X$ such that inequality (2.11) holds. Then $T$ has a unique fixed point.

\section{Acknowledgements}

This research was partially supported by Lorestan University, Khoramabad. 


\section{References}

[1] L. B. Ciric. Generalized contractions and fixed-point theorems Publ. Inst. Math. (Beograd) (N.S.),12(26):19-26, 1971.

[2] L. B. Ciric. Fixed points for generalized multi-valued contractions. Mat. Vesnik, 9(24):265-272,1972.

[3] L. B.Ciric. On fixed points of generalized contractions on probabilistic metric spaces. Publ. Inst.Math. (Beograd) (N.S.), 18(32):71-78, 1975.

[4] A. George and P. Veeramani. On some results in fuzzy metric spaces. Fuzzy Sets and Systems, 64(3):395 \{399, 1994.

[5] M. Grabiec. Fixed points in fuzzy metric spaces. Fuzzy Sets and Systems, 27(3):385\{389, 1988.

[6] V. Gregori and A. Sapena. On fixed-point theorems in fuzzy metric spaces. Fuzzy Sets and Systems, 125(2):245-252, 2002.

[8] D.Mihet. Fuzzy-contractive mappings in non-Archimedean fuzzy metric spaces. Fuzzy Sets and Systems, 159(6):739-744, 2008.

[9] E. Pap, O. Hadzic, and R. Mesiar. A fixed point theorem in probabilistic metric spaces and an application. J. Math. Anal. Appl., 202(2):433-449, 1996.

[10] J. u. Rodriguez Lopez and S. Romaguera. The Hausdorff fuzzy metric on compact sets. Fuzzy Sets and Systems, 147(2):273-283, 2004.

[11] S. Romaguera, A. Sapena, and P. Tirado. The Banach fixed point theorem in fuzzy quasi-metric spaces with application to the domain of words. Topology Appl., 154(10):2196-2203, 2007.

[12] B. Schweizer and A. Sklar. Statistical metric spaces. Pacic J. Math., 10:313-334, 1960.

[13] R. M. Tardi. Contraction maps on probabilistic metric spaces. J. Math. Anal. Appl., 165(2):517-523, 1992.

[7] I. Kramosil and J. Michalek. Fuzzy metrics and statistical metric spaces. Kybernetika (Prague), 11(5):336-344, 1975. 